\title{
Responsabilidad social universitaria, diagnóstico en dependencia de educacion superior
}

\section{University social responsibility, diagnosis in dependence on higher education}

\author{
PÉREZ-BRAVO, Juliał*，VILLARREAL-ALCALDE，Jorge Gabriel， ARELLANO-ROCHA, \\ Francisco Javier y GONZÁLEZ-NERI, Aarón Iván
}

Universidad Autónoma de Querétaro, Cerro de las Campanas S/N, Centro Universitario, Querétaro, Qro. C.P. 76010.

ID $1^{\text {er }}$ Autor: Julia, Pérez-Bravo / ORC ID: 0000-0002-1310-0145, Researcher ID Thomson: N-3319-2018, CVU CONACYT ID: 501992 ID 1 $1^{\text {er }}$ Coautor: Jorge Gabriel, Villarreal-Alcalde / ORC ID: 0000-0003-3101-3683, Researcher ID Thomson: X-3222-
2018, CVU CONACYT ID: 999686

ID $2^{\text {do }}$ CoAutor: Francisco Javier, Arellano-Rocha / ORC ID: 0000-0001-8530-9936, Researcher ID Thomson: X-21202018, CVU CONACYT ID: 953180

ID $3^{\text {er }}$ Coautor: Aarón Iván, González-Neri

DOI: $10.35429 / J E S C .2019 .9 .3 .7 .18$ Recibido: 10 de Julio, 2019; Aceptado 30 de Septiembre, 2019

\begin{abstract}
Resumen
El propósito del presente trabajo fue diagnosticar en el eje de campus responsable, la situación prevaleciente en materia de Responsabilidad Social Universitaria (RSU) en el año 2018, desde la perspectiva de los docentes en una Dependencia de Educación Superior (DES) instalada en el Estado de Querétaro, la metodología utilizada fue estudio de caso con investigación de campo bajo el enfoque cuantitativo con alcance descriptivo, el instrumento utilizado fue una encuesta estructurada con escala de Likert, la cual fue respondida por 28 docentes de diferentes facultades de un total de 284 . Los resultados encontrados fueron que, la DES en estudio es una dependencia que se encuentra en una etapa de avanzado desarrollo en sus prácticas de RSU, toda vez que, el $35 \%$ de sus indicadores se perciben como incumplidos o deficientes y un $(45 \%)$ se perciben como cumpliendo con las características para ser socialmente responsables, se cuenta también con un $20 \%$ de ítems que se perciben como iniciativas medianamente cumplidas y que aún necesitan reforzarse.
\end{abstract}

Responsabilidad social universitaria, Campus responsable, Dependencia de Educación Superior

\begin{abstract}
The purpose of this article was to diagnose in the responsible campus axis, the prevalent situation in the area of Social University Responsibility (SUR) in 2018, from the perspective of teachers in a Higher Education Unit (HEU) settled in the State of Queretaro, the methodology used was a study case with field research under the focus of a descriptive scope, the instrument used was a survey structured with the Likert scale, which was answered by 28 teachers from different Faculties with a total universe of 284. The founded results were that the HEU under the study is a dependence that is found in an advance development on their SUR practices, due to $35 \%$ of their indicators, were seen as not accomplished of deficient and a $45 \%$ were seen as being accomplished with the characteristics of being socially responsible, it is count that $20 \%$ of items were seen as initiatives half accomplished and that they have to do better.
\end{abstract}

Social university Responsibility, Campus responsibility, Unit of Higher Education

Citación: PÉREZ-BRAVO, Julia, VILLARREAL-ALCALDE, Jorge Gabriel, ARELLANO-ROCHA, Francisco Javier y GONZÁLEZ-NERI, Aarón Iván. Responsabilidad social universitaria, diagnóstico en dependencia de educacion superior. Revista de Ciencias de la Educación. 2019. 3-9: 7-18

\footnotetext{
* Correspondencia del Autor (correo electrónico: jpb448@ hotmail.com)

$\dagger$ Investigador contribuyendo como primer autor.
} 


\section{Introducción}

Actualmente, el estudio de la responsabilidad social en las universidades es aún limitado y de carácter descriptivo, por lo que, de inicio, para posteriormente ir ahondando mayormente, se centran en las percepciones parciales de los estudiantes, docentes o autoridades (Montgomery \& Ramus, 2003); o en algunos aspectos curriculares (Gordon, \& Gelardi, 2005). Aunque con Vallaeys (2009), se inician trabajos sobre sistemas de gestión o de aplicación y evaluación de modelos de responsabilidad social, al igual que con el mismo investigador se inician estudios sobre metodologías de medición y evaluación en el año 2014.

La presente investigación, también se centra sobre percepciones de stakeholders internos (docentes), surgiendo como hipótesis de interés las siguientes: ¿Cuál es la delimitación teórica de la responsabilidad social universitaria?, ¿Cuáles son las percepciones de los stakeholders internos (docentes) respecto de las acciones de responsabilidad social universitaria y cómo afecta su compromiso y satisfacción con la universidad?

Estos cuestionamientos son de particular interés para quien esto escribe y aunque, las autoridades universitarias ya están sensibilizados en la materia y trabajando en lo concerniente, es necesario contar con datos que, de la presente investigación surjan y que pueden servir de guía para bosquejar e implantar mejoras en el sistema de gestión de responsabilidad social al área específica que aquí se plantea.

Dicho lo anterior, para la presente investigación se plantea el siguiente:

\section{Objetivo}

El propósito del presente trabajo fue diagnosticar en el eje de campus responsable, la situación prevaleciente en materia de Responsabilidad Social Universitaria (RSU) en el año 2018, desde la perspectiva de los docentes en una Dependencia de Educación Superior (DES) instalada en el Estado de Querétaro.

\section{Enmarcamiento teorico}

\section{Antecedentes}

Desde una perspectiva global y gracias a las tecnologías de la información hoy se puede ingresar a vastos datos generados a través del tiempo sobre responsabilidad social, desde diversos enfoques, de diferentes autores y desde diversos puntos geográficos en nuestro planeta, aunque los principales estudios sobre la RS versan principalmente sobre la responsabilidad social corporativa.

Este tema ha despertado el interés de estudiosos dedicados a las áreas sociales y de aquellos de otras áreas, que han identificado la magnitud de la importancia de desempeñarse socialmente responsable en su ámbito de trabajo, así se observa que hay responsabilidad social en las corporaciones de todo tipo y tamaño, las tecnologías, la ingeniería, la medicina, la abogacía, en fin, en el ejercicio de todas y cada una de las profesiones.

Hoy se puede conocer desde los antecedentes de la Responsabilidad Social Corporativa (RSC), enmarcados en la visión de diversos autores como de la cuesta (2005), quien la contextualizó en los años 20 como acción corporativa de una empresa, no como una acción filantrópica de un solo empresario, otros autores afirman que surge en los años cincuenta ligándola con el crecimiento rápido de las empresas americanas y a la importancia que toman para la sociedad, otros como Gilli (2012), la llevan a 1960 cuando se plantea la cuestión en los términos actuales y se exige que la empresa asuma la responsabilidad por los problemas sociales y que contribuya a su solución, aunque hay quienes van más atrás como Peraza (2014), y desde el punto de vista epistemológico la sitúa en la época de la edad media, ya que ésta nace cuando se establecen reglas de conducta, de urbanismo y de valores éticos y morales para la convivencia del hombre en sociedad o comunidad. De igual forma se puede dar cuenta de los organismos internacionales y documentos realizados, los cuales han participado de manera muy importante, en diversos tiempos y que han sentado las principales bases en materia de responsabilidad social, los cuales con el transcurso del tiempo han proporcionado, un amplio material de referencia para conocer e implementar acciones de RSC en cualquier organización, así se tienen, por citar algunos a: 
La Organización para la Cooperación y el Desarrollo Económico (OCDE), donde en 1976, se elaboró la primera versión de las líneas directrices para las empresas multinacionales, las cuales se revisan nuevamente en los años 2000 y 2011, agregando la dimensión del consumo sostenible como una nueva responsabilidad.

La Organización Internacional del Trabajo (OIT), donde se aprueba en 1977 la Declaración Tripartita de Principios sobre las Empresas Multinacionales y la Política Social, abarcando los temas de: condiciones de trabajo y vida, relaciones de trabajo, empleo y formación.

La Caux Round Table fundada en 1986, la cual centra su interés en las relaciones económicas y sociales constructivas entre los países miembros y sus responsabilidades.

La creación en 1997 de la iniciativa del reporte global (GRI), en donde se desarrolló la Guía para la elaboración de un informe de sostenibilidad de las empresas.

La Cumbre de Lisboa en marzo del 2000, en la cual se abordaron, entre otros temas, cuestiones sociales a favor de las personas en situaciones de pobreza y exclusión social.

La publicación del Libro verde de la responsabilidad social empresarial de la Comunidad Europea en 2001, que establece lineamientos éticos, sociales y medioambientales para las empresas.

La elaboración y firma del Global Compact (Pacto Mundial), por iniciativa de la Organización de Naciones Unidas (ONU) con lo cual se crea el marco que permitiría la gestión de la RSC en las empresas en un entorno global y bajo valores éticos universales, dando inicio en el Foro Económico Mundial en 1999, tomando forma definitiva en 2000 y actualizándose en 2004, donde se agregó como pilar de defensa la lucha contra la corrupción plasmado en el décimo principio.

La Organización Internacional de Estandarización (ISO) que, en 2005 implementa el proyecto de normalización global ISO 26000 en el campo de la responsabilidad social.
Los organismos anteriores y el acervo generado, induce a conocer sobre los principios básicos rectores a manera de normas, guías, recomendaciones o directrices, las cuales coinciden en que las principales vertientes o temas a considerar, cuando se implementa la RSC en una organización, son los derechos humanos, estándares laborales, medio ambiente, anti-corrupción y el consumo sostenible.

Nuestro país no se queda atrás, dándose inicio a la institucionalización y promoción del movimiento de responsabilidad social en Mayo de 2000, al celebrarse el Tercer Congreso de Responsabilidad Social de las Américas, aunque podemos decir que ya se trabajaba en la materia desde la conformación del Centro Mexicano para la Filantropía (CEMEFI) en 1998, mismo que se fortalece con la aparición de la Alianza para la responsabilidad Social Empresarial (AliaRSE) en el año 2001 y actualmente son los organismos promotores y certificadores en el país, los cuales han logrado que las empresas en México se interesen en la materia e inicien acciones diversas para ser socialmente responsables.

Actualmente se encuentran operando en México en materia de responsabilidad social asociaciones empresariales, instituciones académicas organismos internacionales, organismos públicos, organizaciones de sociedad civil y organizaciones de responsabilidad social empresarial, con diversas líneas de acción tales como: Articulación, asesoramiento, capacitación, divulgación, estandarización, financiamiento, investigación y reconocimiento, pudiendo estar en uno o varios de los siguientes dominios: De consumidores y clientes, gobierno empresarial, inversión social, medio ambiente, prácticas laborales, proveedores y responsabilidad social empresarial general. Lo cierto es que la RSC ha logrado permear en todo el mundo, principalmente en las empresas de carácter privado, cuyos directivos han logrado o están gestionando el aprovechar en su beneficio, las bondades de ser socialmente responsable en sus prácticas y, México, inmerso en el mundo de la competitividad, obligado por la globalidad, no ha sido la excepción, encontrándose así empresas ya trabajado al cien por ciento y con distintivos nacionales e internacionales, presentando informes de empresas socialmente responsables $\mathrm{o}$ en diversos niveles de los implementación de la RSC al interior de la organización.

PÉREZ-BRAVO, Julia, VILLARREAL-ALCALDE, Jorge Gabriel, ARELLANO-ROCHA, Francisco Javier y GONZÁLEZ-NERI, Aarón Iván. Responsabilidad social universitaria, diagnóstico en dependencia de educacion superior. Revista de Ciencias de la Educación. 2019 


\section{Y, ¿Qué pasa con las instituciones educativas en materia de responsabilidad social?}

De acuerdo con Montalvo, Villanueva, Armenteros y Cervantes (2016), en México, siguiendo las acciones del Centro Mexicano para la Filantropía (CEMEFI) y los principios del Pacto Mundial en materia de responsabilidad social corporativa, las universidades, de acuerdo a sus áreas prioritarias, definen su propio modelo o estructura de RSU para integrarla a los objetivos institucionales, siguiendo el uso del uso del Manual de Vallaeys, De la Cruz, and Sasia (2009) difundido por el Banco Internacional de Desarrollo (BID) y Asociación Mexicana de RSU.

Y aunque la responsabilidad social es un tema actualmente conocido, principalmente en el mundo de los negocios, las investigaciones sobre el tema aplicado en el mundo educativo son, relativamente nuevas y aún están en progreso y aumentando a grandes pasos en América Latina y México, como lo muestran los esfuerzos realizados por: (entre otros organismos gubernamentales y no gubernamentales), la Unión de responsabilidad social universitaria latinoamericana (URSULA), en donde confluyen diferentes actores del desarrollo, en aras de discutir profundamente en torno al rol de la universidad y de las distintas estrategias y metodologías para llevarlo a cabo de manera efectiva.

Lo anterior también se puede observar al encontrar que las investigaciones en materia de responsabilidad social universitaria han aumentado en un $500 \%$, de acuerdo con Baca (2015), quien cita a Gordon \& Gelardi (20059 y De la Cuesta \& Sánchez, (2011), entre otros autores, y menciona al respecto, que las universidades se han convertido en un foco de interés en esta materia y son un campo fructífero de investigación.

\section{¿Cómo se define la Responsabilidad social universitaria?}

La Responsabilidad Social Universitaria (RSU) se ha definido como "una nueva política de gestión universitaria que se va desarrollando en Latinoamérica para responder a los impactos organizacionales y académicos de la universidad.
Se distingue tanto de la tradicional extensión solidaria como de un mero compromiso unilateral declarativo y obliga a cada universidad a poner en tela de juicio sus presupuestos epistémicos y su currículo oculto. Como tal, la RSU no es cómoda, puesto que fuerza a la autocrítica institucional" (Vallaeys, François, 2014).

Vallaeys, François (2009), la autoridad en la materia en América Latina más reconocida por los trabajos que realiza en pro de la responsabilidad social, establece los ámbitos o impactos, dimensiones y ejes siguientes para la RSU:

Cuatro ámbitos de actuación o impacto de la universidad

1. Ámbito organizacional: en tanto institución que opera en torno a un proyecto universitario, con una estructura que lo desarrolla y unas políticas concretas que lo promueven. Una institución, además, que consume, contrata, genera residuos, etcétera.

2. Ámbito educativo: en tanto institución que se encarga de la formación de sus estudiantes, con una vocación profesionalizante y cívica.

3. Ámbito del conocimiento: en tanto institución que investiga, que produce saber y lo transmite.

4. Ámbito social: en tanto institución que forma parte de la sociedad e interactúa con otros agentes, colectivos y comunidades, tanto a nivel local como global.

También establece los stakeholders, partes interesadas o grupos de interés.

1. Personal no docente

2. Personal docente investigador

3. Autoridades

4. Estudiantes

5. Proveedores

6. Egresados

7. Empleadores

8. Competidores

9. Comunidades locales

10. Organizaciones socias

11. Estado. 
Así como los cuatro ejes de implicación de la misma RSU, que se plantean también como el deber hacer de una institución educativa a nivel superior

1. Campus responsable: implica la gestión socialmente responsable de la organización y sus procedimientos institucionales; del clima laboral, el manejo de los recursos humanos, los procesos democráticos internos $\mathrm{y}$ el cuidado del medio ambiente.

2. Formación profesional y ciudadana: es la gestión socialmente responsable de la formación académica (en su temática, organización curricular, metodología y propuesta didáctica).

3. Gestión social del conocimiento: es la gestión socialmente responsable de la producción y difusión del saber, la investigación y los modelos epistemológicos promovidos desde el aula. Es decir, orientar la actividad científica, concertando líneas e investigación con partes interesadas externas para que se produzca conocimiento acorde a la agenda de desarrollo local y nacional y programas sociales del sector público.

4. Participación social: es la gestión socialmente responsable de la participación de la universidad en la comunidad. Es decir, realizar proyectos con otros actores creando vínculos para el aprendizaje mutuo y el desarrollo social.

\section{Metodología}

En la presente investigación la metodología utilizada fue, estudio de caso con investigación de campo bajo el enfoque cuantitativo con alcance descriptivo. En esta primera parte de la investigación, se usa una aproximación cuantitativa para obtener información estadística sobre la percepción que tienen los docentes como uno de los principales stakeholders internos en el apartado concerniente a campus responsable respecto de las diversas actividades de RSU que se vienen realizando en la institución. Esta información es obtenida de la aplicación del instrumento consistente en una encuesta estructurada con escala de Likert, aplicada a este grupo de interés cuya muestra consistió en 23 docentes de diferentes facultades de un total de 284 .
Aunque en la investigación mayor, de la cual forma parte el presente trabajo, también se usará una aproximación cualitativa para obtener información detallada sobre las diversas acciones y gestiones de RSU que viene realizado la universidad, información que procederá tanto desde informantes clave como del análisis del contenido de documentos de gestión públicamente accesibles (Ejemplo: Memorias institucionales sobre filosofía, planes, políticas, manuales, códigos, informes y reportes de gestión, boletines informativos) así como de la revisión de su página web (www.uaq.mx).

\section{Muestra}

Para analizar la situación de la RSU de la Universidad Autónoma de Querétaro, específicamente en la DES San Juan del Río, se trabajó con grupo de stakeholders internos que proporcionó la información según la lista de chequeo de RSU utilizada para tal fin y dada la oportunidad de estar participando en un curso taller de políticas públicas y RSU y la disponibilidad y consentimiento de los participantes, la muestra representativa la conformaron los 23 participantes a quienes se encuestó en su totalidad.

\section{Instrumentos}

Se ha utilizado un instrumento de investigación consistente en un cuestionario auto-aplicado de escala Likert, denominado ejercicio de autodiagnóstico de percepciones, elaborado por François Vallaeys, Cristina de la Cruz y Pedro M. Sasia (2009), para estimar en qué medida, la institución cumple con cada indicador de responsabilidad social universitaria. Este instrumento incluye los 75 ítems o indicadores, seleccionándose para el presente trabajo solo los 20 ítems de RSU (CR1-CR20), que representarían contacto directo con los stakeholders internos en la dimensión campus responsable, presentados como preguntas o afirmaciones de lo que la universidad realiza en cuanto a RSU que van desde: Totalmente en desacuerdo,

En desacuerdo, Parcialmente en desacuerdo, Parcialmente de acuerdo, De acuerdo, hasta Totalmente de acuerdo y, que determinarán el nivel de aceptación y percepción del docente con las diversas acciones de RSU de la institución y/o qué tan presente está en la vida laboral del personal docente. 


\section{Resultados}

De los resultados obtenidos, se presenta una síntesis de la información obtenida mediante la encuesta, en la dimensión campus responsable, quedando pendiente por analizar las restantes tres dimensiones: Formación profesional y ciudadana, Gestión social del conocimiento y Participación social (Proyectos emprendidos en comunidad docentes de extensión). Así mismo se muestran en forma gráfica con el porcentaje de indicadores de la citada dimensión (20 ítems de CR1 a CR20) según su nivel de cumplimiento para la DES en estudio.

Se observó, de acuerdo a los resultados obtenidos que, en general, la Universidad Autónoma de Querétaro específicamente, la DES en estudio, es una institución en etapa mediana de desarrollo en materia de RSU en la dimensión de campus responsable, toda vez que se pudo encontrar que, de los 20 ítems de percepción de campus responsable, 10 fueron positivos en la percepción de los docentes (50\%), siendo los siguientes:

Ítem CR1: Satisfacción con nivel de remuneración

Ítem CR5: Nivel de percepción sobre la existencia de facilidades para el desarrollo personal y profesional de los profesores.

Ítem CR6: Nivel de percepción sobre la existencia de equidad de género en las instancias de gobierno en la Universidad.

Ítem CR7: Percepción sobre la no existencia de discriminación en el acceso a la docencia, ni por género, religión, raza, orientación sexual o política

Ítem CR8: Percepción sobre si la universidad es socialmente responsable con su personal docente y no docente

Ítem CR13: Percepción respecto de, si las autoridades de la universidad han sido elegidas en forma democrática y transparente

Ítem CR17: Percepción sobre la coherencia entre los principios que declara la universidad y lo que se practica en el campus
Ítem CR18: percepción respecto de si, la comunicación y el marketing de la universidad se llevan a cabo en forma socialmente responsable

Ítem CR19: Percepción respecto de, si la universidad promueve relaciones de cooperación con otras universidades del medio

Ítem CR20: Percepción sobre si, la universidad busca utilizar sus campañas de marketing para promover valores y temas de responsabilidad social.

Los siguientes 06 fueron negativos, es decir un 30\% del total de los ítems:

Ítem CR9: percepción sobre si la universidad es ambientalmente responsable

Ítem CR10: percepción sobre la existencia de una política institutional para la protección del medio ambiente en el campus

Ítem CR11: Percepción sobre sobre la existencia de capacitación en temas ambientales por parte de la universidad para el personal docente y no docente

Ítem CR12: Percepción sobre si la organización en el campus, permite a las personas adquirir hábitos ecológicos adecuados

Ítem CR14: percepción sobre, si me siento escuchado como ciudadano y puedo participar activamente en la vida institucional

Ítem CR16: Percepción respecto de si, se brinda periódicamente información económicofinanciera al personal de la Universidad.

Se puede considerar que se mantienen neutrales o en la media, por así decirlo, 04 de ellos, representando un $20 \%$ del total:

Ítem CR2: Nivel de aceptación respecto de la satisfacción con los beneficios sociales y profesionales que brinda la universidad

Ítem CR3: percepción sobre la promoción del trabajo en equipo y la solidaridad

Ítem CR4: percepción sobre la existencia de un buen clima laboral entre el personal 
Ítem CR15: percepción sobre si la Universidad informa adecuadamente acerca de todas las decisiones institucionales que conciernen y afectan a los docentes.

No obstante, cabe mencionar que estos resultados pueden verse influenciados por distintos factores ya que la muestra fue heterogénea y los docentes encuestados no pertenecen a una sola facultad, a un mismo tipo de contratación o, a un mismo nivel de grado de preparación.

De igual forma se debe considerar que, el evento en el que se encontraban participando los docentes, formó parte de las primeras acciones tomadas por el gobierno de la institución en materia de RSU (la concientización y capacitación), por lo que los resultados pueden hoy percibirse como diferentes a un año de haberse realizado la investigación, tiempo en el que las acciones de la institución en estudio, en materia de RSU, se han reforzado por iniciativa del gobierno institucional por interés propio y en seguimiento a lo plasmado en los Objetivos de Desarrollo del Milenio (ODS).

A continuación, se presentan los resultados de forma gráfica para su mejor apreciación.

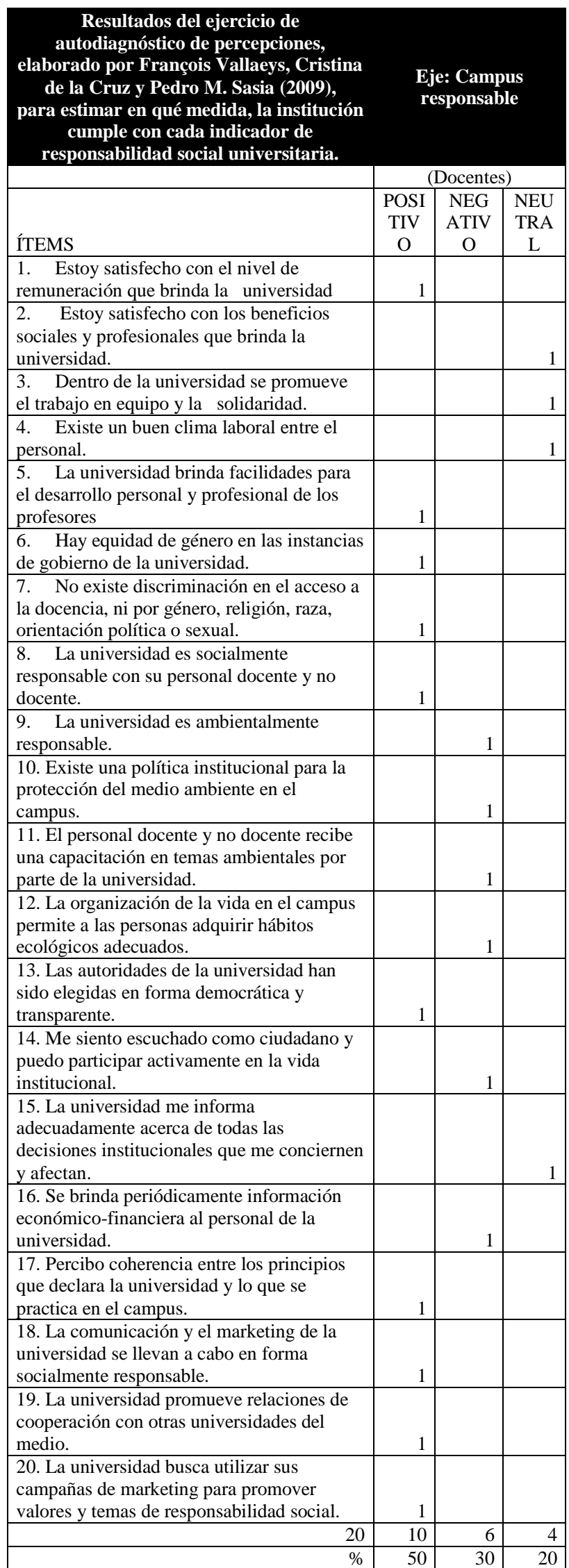

Tabla 1 Concentrado de resultados del ejercicio de diagnóstico de percepciones sobre en qué medida, la institución cumple con cada indicador de responsabilidad social universitaria en el eje Campus responsable para docentes.

Fuente: Elaboración Propia 
Por cuestión de espacio, se presentan únicamente los gráficos realizados para los ítems en los que el resultado es por demás llamativo debido al nivel de insatisfacción o satisfacción que representan, o a los niveles de percepción positiva o negativa que de igual forma se presenta en los encuestados para el eje de campus responsable.

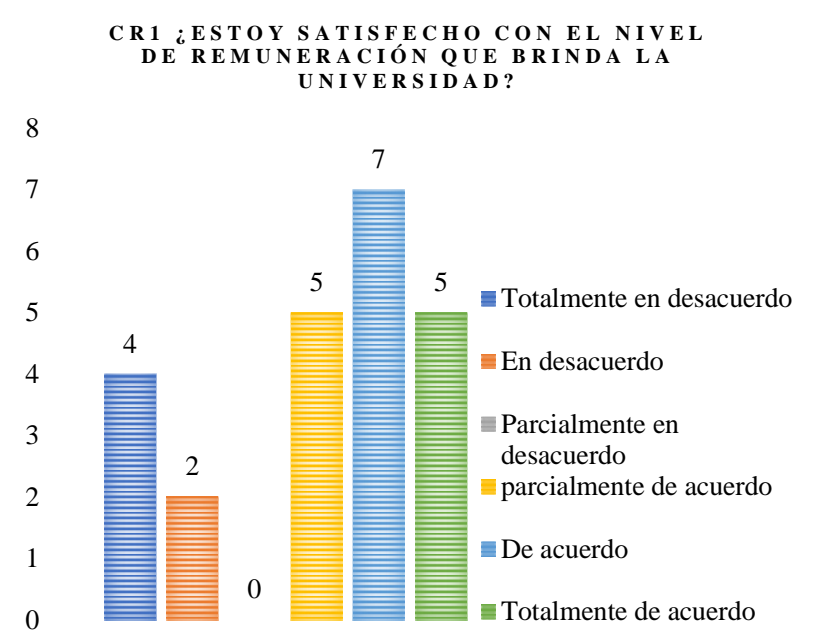

Gráfico 1 Nivel de aceptación respecto de la satisfacción con el nivel de remuneración

Fuente: Elaboración Propia

Un $21.74 \%$ de "totalmente de acuerdo" más el 30.43\% que representa el "de acuerdo", sumando un $52.17 \%$ dan cuenta de que los docentes se encuentra satisfecho con el nivel de remuneración que le brinda la Universidad

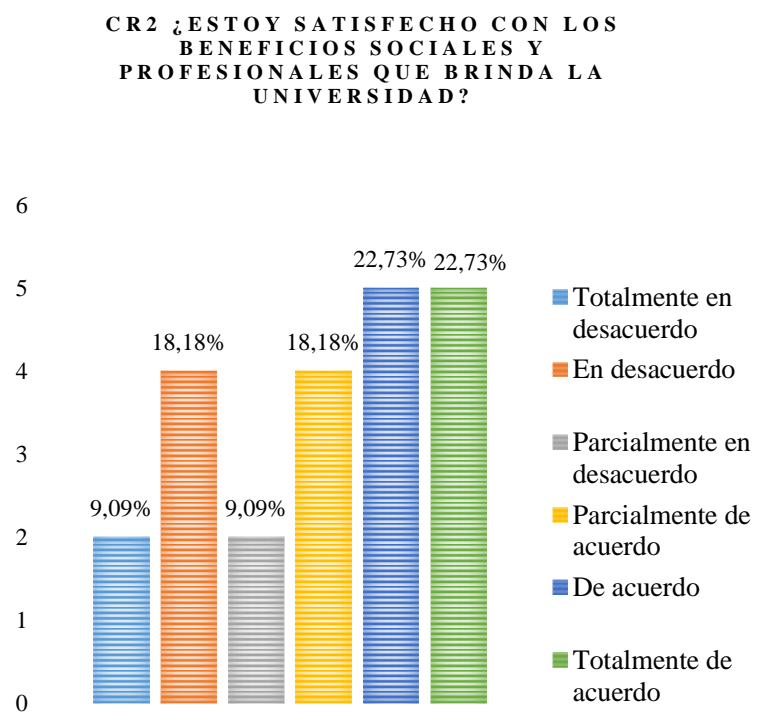

Gráfico 2 Nivel de aceptación respecto de la satisfacción con los beneficios sociales y profesionales que brinda la universidad

Fuente: Elaboración Propia
De igual forma la suma del "totalmente de acuerdo" con la del "de acuerdo" dan un $45.46 \%$ de los docentes que se encuentran satisfechos con los beneficios sociales y profesionales que brinda la universidad.

CR5 CLA UNIVERSIDAD BRINDA FACILIDADES PARA EL DESARROLLO PERSONAL Y PROFESIONAL DE LOS PROFESORES?

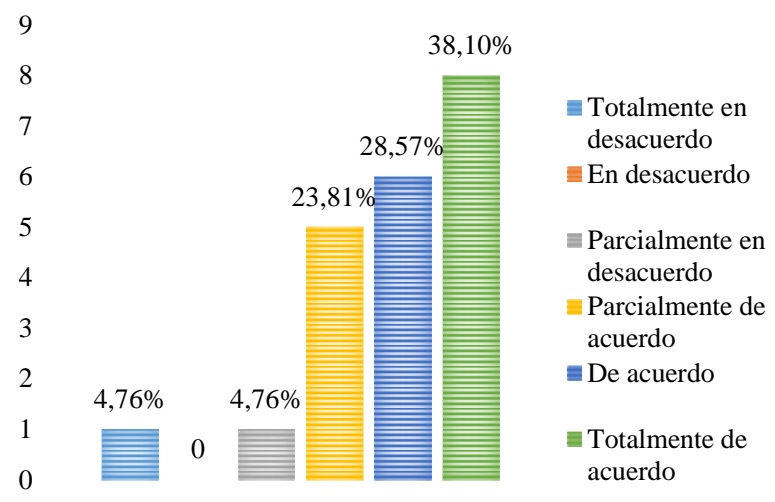

Gráfico 3 Nivel de percepción sobre la existencia de facilidades para el desarrollo personal y profesional de los profesores

Fuente: Elaboración Propia

Resulta claro que es negativa la percepción sobre sobre la existencia de capacitación en temas ambientales por parte de la universidad para el personal docente y no docente, es decir, no existe, lo cual se muestra con un $45.46 \%$.

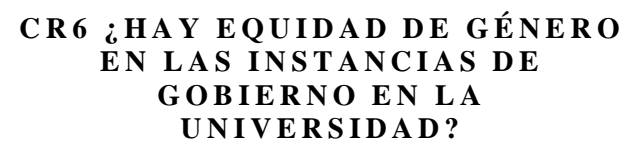

8

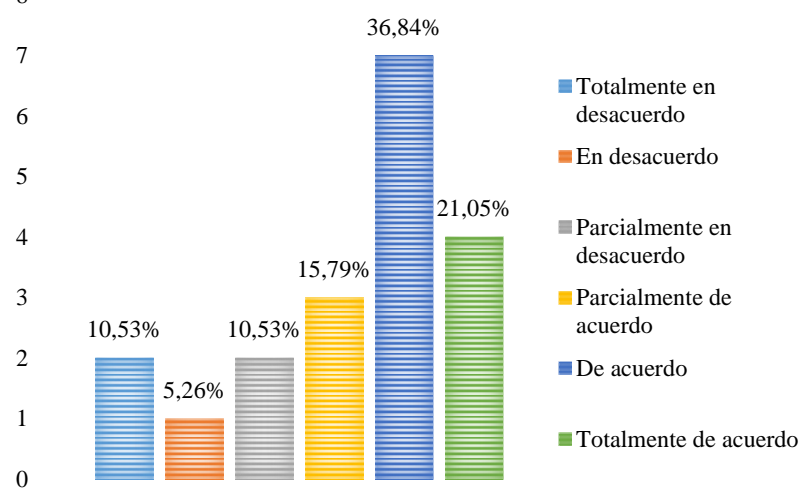

Gráfico 4 Nivel de percepción sobre la existencia de equidad de género en las instancias de gobierno en la Universidad

Fuente: Elaboración Propia 
De igual forma, los más elevados porcentajes, sumando un $66.67 \%$ muestran que la percepción sobre la existencia de facilidades para el desarrollo personal y profesional de los profesores es muy alta.

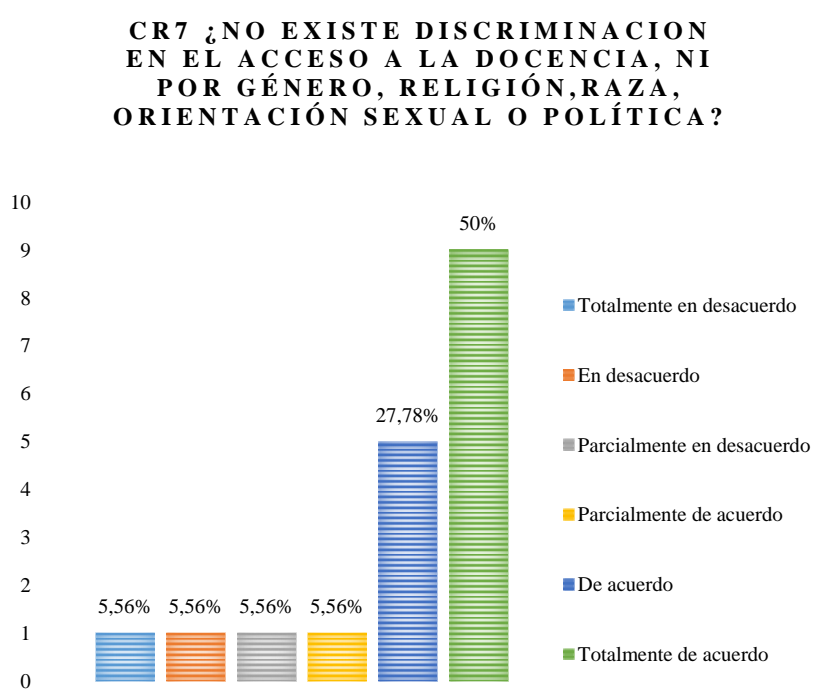

Gráfico 5 Percepción sobre la no existencia de discriminación en el acceso a la docencia, ni por género, religión, raza, orientación sexual o política Fuente: Elaboración Propia

Es contundente la afirmación de que se percibe la existencia de equidad de género en las instancias de gobierno en la Universidad con un $57.89 \%$

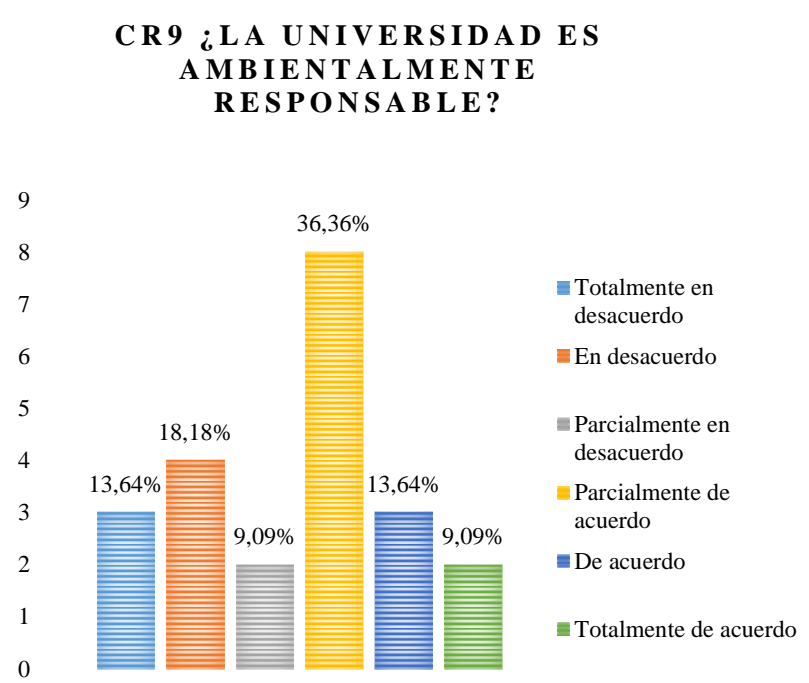

Gráfico 6 percepción sobre si la universidad es ambientalmente responsable

Fuente: Elaboración Propia

En cuanto a la no existencia de discriminación en el acceso a la docencia, ni por género, religión, raza, orientación sexual o política muestra un contundente $77.78 \%$

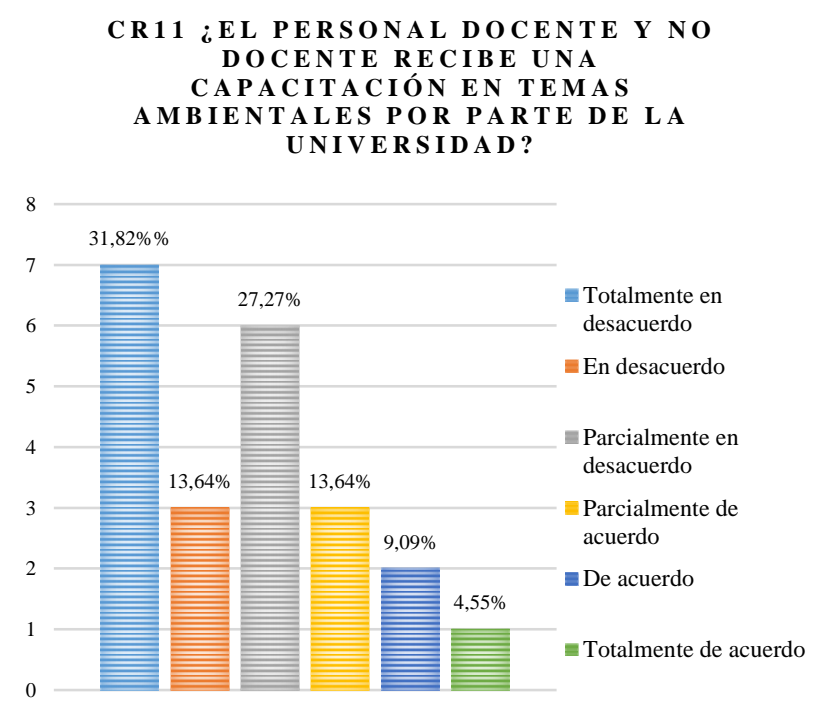

Gráfico 7 Percepción sobre sobre la existencia de capacitación en temas ambientales por parte de la universidad para el personal docente y no docente.

Fuente: Elaboración Propia

En este rubro, el mayor porcentaje fue para un "parcialmente de acuerdo", seguido de "en desacuerdo" y" totalmente en desacuerdo", lo cual da muestra de una percepción negativa sobre si la institución es ambientalmente responsable.

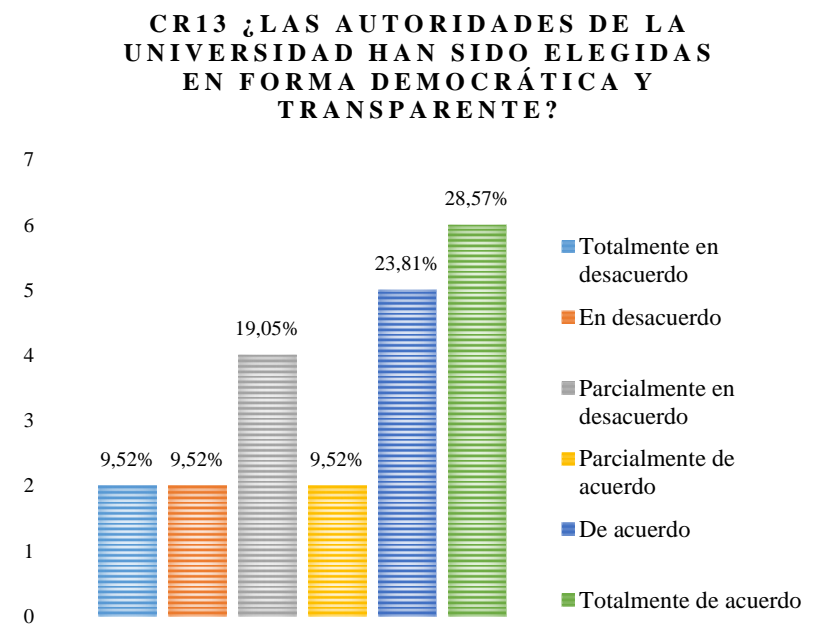

Gráfico 8 Percepción respecto de, si las autoridades de la universidad han sido elegidas en forma democrática $\mathrm{y}$ transparente

Fuente: Elaboración Propia

Resulta contundente la percepción positiva sobre la existencia de democracia y transparencia en la elección de las autoridades universitarias con un $52.38 \%$ de percepción positiva. 


\section{Discusión}

Utilizando los instrumentos comentados en el apartado de metodología, se ha evaluado el nivel de RSU en la DES San Juan del Río y en general, se observó, de acuerdo a los resultados obtenidos que, la DES en estudio, es una institución en etapa mediana de desarrollo en materia de RSU en la dimensión de campus responsable, toda vez que se pudo encontrar que, de los 20 ítems de percepción de campus responsable, 10 fueron positivos en la percepción de los docentes (50\%), 06 fueron negativos, es decir un 30\% del total y, 04 de ellos se mantuvieron en un término medio o neutrales, representando un $20 \%$.

Lo cual concuerda con los resultados encontrados por la Investigación Continental URSULA: Estado del arte de la Responsabilidad Social Universitaria (RSU) en América Latina 2018, en la cual participaron auto diagnosticándose 12 universidades de México, donde se afirma que las 60 universidades de los 9 países participantes tienen aún mucho que avanzar, afirman que se encontraron buenos promedios en la gestión interna del clima laboral y la ética-transparencia-inclusión, y que contrastan con los puntos débiles en gestión ambiental interna.

De lo dicho, se desprende que la DES puede y debe seguir avanzando en su nivel de maduración de RSU, considerando además que recientemente se han implementado diversos programas e iniciativas internacionales $y$ nacionales que se deben seguir a efecto de acatar sus recomendaciones en beneficio de la sociedad atendida por las instituciones educativas, tales como los objetivos del milenio (Agenda 2030), y los que se desprenden del Plan Nacional de Desarrollo en vigor y las propuestas por la Asociación Nacional de Universidades e Instituciones de Educación Superior (ANUIES) y la Unión de Responsabilidad Social Universitaria Latinoamericana (URSULA).

Como limitantes en el presente estudio, se encuentra que, solo se ha realizado con stakeholders internos (docentes), sin una muestra representativa de los otros actores internos (dirigentes, estudiantes y trabajadores, así como externos de la universidad, tales como empleadores, empresas proveedoras, clientes, gobierno, municipalidades, sociedad civil y otras entidades y personas que tengan vinculación directa o indirecta con la DES.

\section{Referencias}

AECA (2004) Marco Conceptual de la Responsabilidad Social Corporativa. Madrid, España.

Andreu (2005) LA responsabilidad Social Corporativa; UN concepto por definir. En Cierec-España, revista de economía pública, social y cooperativa, noviembre 2005 , numero 053.

Baca Neglia, Hilda Zoraida (2015) LA RESPONSABILIDAD SOCIAL UNIVERSITARIA: PROPUESTA CONCEPTUAL Y MEDICIÓN EN EL ÁMBITO DE UNA UNIVERSIDAD PRIVADA DE LIMA - PERÚ 2015

Comisión de las Comunidades Europeas, LIBRO Verde (2007). Fomentar un marco europeo para la responsabilidad social de las empresas. Bruselas: Comisión de las Comunidades Europeas. Obtenido: 27 de diciembre de 2011, desde http://www.jussemper.org/Inicio/Recursos/Acti vidad\%20Corporativa/Resources/Libro\%20ver de.pdf.

Correa, Sharon Flynn y Alon Amit (2004). RESPONSABILIDAD social corporativa en América Latina: UNA visión empresarial. Serie: Medio ambiente y desarrollo, núm. 85. CEPAL: Santiago de Chile. Disponible en internet: Obtenido 27 de diciembre de 2011. Desde http://www.eclac.org/publicaciones/xml/4/1490 4/lcl2104.pdf.

CEOE (2011) Responsabilidad Social Corporativa. Libro Blanco de la RSC. Disponible en: http://www.ciberoamericana.com/pdf/Guia1_R SC.pdf

CSR Europe (2002) It simply works better! Informe de la Campaña Europea sobre RSE. The Copenhagen Centre. Disponible en: http://www.sequilibre.com/herramientas/CAM_ Spanish_It_simply_works_better.pdf (Recuperado en Agosto 8, 2015)

De la Cuesta, M. (2011). Responsabilidad Social Universitaria. Madrid: Fundación Carolina. 
Domínguez (2005) X Dedalo consultores, LA responsabilidad social corporativa, UNA historia reciente, Obtenido el 28 de diciembre de 2013 desde http://www.dedaloconsultores.com/boletin/bole tin001/articulo_RSC.pdf.

Duran, A. y Giraldo, E. (2004) El reto de la acreditación progresiva. Aplicación a herramientas de Gestión de la Responsabilidad Social Corporativa. VIII Congreso de Ingeniería de Organización Leganés. Recuperado de: http://www.researchgate.net/publication/45448 444_El_reto_de_la_acreditacin_progresiva._Ap licacin_a_herramientas_de_Gestion_de_la_Res ponsabilidad_Social_Corporativa (Consultado en Agosto 8, 2015)

Fernández García, Dr. F. (2009) Responsabilidad social corporativa. España: Editorial Club Universitario

Fundaliza (2008), RESPONSABILIDAD Social Empresarial en chile. Obtenido http://www.alafec.unam.mx/docs/mem_prog_cr /TrabajosInvestigacion/TareaPrevia/Responsabi lidadSocEmprChileGR02.pdf.

Gordon, I., \& Gelardi, M. (2005). Factors That Affect Understanding of Social Responsibility Accounting. Canadian Accounting Perspectives, $4(1), 31-59$.

https://www.iadb.org/es/acerca-delbid/perspectiva-general

https://www.oecd.org/centrodemexico/laocde/la ocdeenmexico.htm

https://www.ilo.org/global/lang--en/index.htm

https://www.globalreporting.org/Pages/default.a $\operatorname{spx}$

https://www.undp.org/content/undp/es/home/su stainable-development-goals.html

Libro Blanco sobre responsabilidad ambiental, COM (2000) 66 final, 9 de febrero de 2000, a través de http://ec.europa.eu/environment/legal/liability/p df/el_full_es.pdf
Lizcano, J.L. y Nieto, P. (2005) ¿Responsabilidad social corporativa o solo corporativa? Asociación Española de Contabilidad y Administración de Empresas.

López Martínez Gema (2010) "LA REALIDAD ESPAÑOLA DE LA RSC A TRAVÉS DE LOS INFORMES DE SOSTENIBILIDAD DE LAS EMPRESAS EN EL BIENIO 2006-2007” Tesis para obtener el grado de Doctor en ciencias de la información, Universidad complutense de Madrid. Madrid, 2010.

Montalvo Morales Jesús Alberto, Villanueva Armenteros Yanet, Armenteros Acosta Maria del Carmen y Cervantes Ávila Yazmín Guadalupe (2016), LA RESPONSABILIDAD SOCIAL AUTODIAGNÓSTICO DE LA EFECTIVIDAD DE LA RELACION UNIVERSIDAD SOCIEDAD EN LA UNIVERSIDAD ESTATAL DE COAHUILA, MÉXICO, recuperado de: http://www.aeca1.org/pub/on_line/comunicacio nes_xviiicongresoaeca/cd/158c.pdf

Montgomery, D., \& Ramus, C. (2003). Corporate social responsibility reputation effects on MBA job choice. Research Paper No. 1805, Stanford Graduate School of Business.

Observatorio de la RSC (2005) Guía de la RSC para las Pymes. Disponible en: http://observatoriorsc.org/wpcontent/uploads/2013/07/Guia_RSC_PYME.pd $\mathrm{f}$

Organización Internacional de Estandarización. 2010. ISO 26000 Responsabilidad Social. A través de http://www.iso.org/iso/discovering_iso_26000es.pdf. Recuperado en marzo de 2014.

Oxfam International, a través de https://www.oxfam.org/es

Pacto mundial o global compact a través de: https://www.unglobalcompact.org/

Unión de responsabilidad social universitaria latinoamericana (URSULA), a través de: http://unionursula.org/ 
Unión de Responsabilidad Social Universitaria Latinoamericana [URSULA] (2018). Investigación Continental URSULA: estado del arte de la Responsabilidad Social Universitaria (RSU) en América Latina - 2018. Recuperado de: http://unionursula.org/investigacioncontinental-rsu2018/

Vallaeys, François. (2014). La responsabilidad social universitaria: un nuevo modelo universitario contra la mercantilización. Revista iberoamericana de educación superior, 5(12), 105-117. Recuperado en 21 de enero de 2019, de http://www.scielo.org.mx/scielo.php?script=sci _arttext\&pid=S2007-

$28722014000100006 \& \operatorname{lng}=$ es\&tlng=es.

Vallaeys, François, De la Cruz Cristina y Sasia Pedro M. (2009), Responsabilidad social universitaria: manual de primeros pasos, McGraw-Hill interamericana editores, s.a. de c.v., Derechos reservados (C) Banco Interamericano de Desarrollo, 2009. www.iadb.org 